\title{
Correction to: Relationship between cognitive flexibility and subsequent course of mood symptoms and suicidal ideation in young adults with childhood-onset bipolar disorder
}

Heather A. MacPherson ${ }^{1,2}$ (D) . Anastacia Y. Kudinova ${ }^{1,2}$. Elana Schettini ${ }^{1}$. Gracie A. Jenkins ${ }^{1}$. Anna C. Gilbert ${ }^{1}$. Sarah A. Thomas ${ }^{1,2} \cdot K$ erri L. Kim ${ }^{1,2} \cdot$ Petya D. Radoeva ${ }^{1,2} \cdot$ Rebecca L. Babcock Fenerci $^{1,2} \cdot$ Shirley Yen $^{2,3}$. Heather Hower ${ }^{2,4,5}$. Jeffrey Hunt ${ }^{2}$ - Martin B. Keller ${ }^{2}$ - Daniel P. Dickstein ${ }^{1,2}$

Published online: 18 March 2021

(c) Springer-Verlag GmbH Germany, part of Springer Nature 2021

\section{Correction to: European Child \& Adolescent Psychiatry https://doi.org/10.1007/s00787-020-01688-0}

The original article was published with incorrect funding information. The correct funding information is R01MH087513 (PI: Dickstein), R01MH59691 (PIs: Keller/Yen), and R01MH112543 (PI: Yen).

The original article has been corrected.

The original article can be found online at https://doi.org/10.1007/ s00787-020-01688-0.

Heather A. MacPherson

heather_macpherson@brown.edu

1 Pediatric Mood, Imaging, and NeuroDevelopment (PediMIND) Program, Emma Pendleton Bradley Hospital,

East Providence, RI, USA

2 Department of Psychiatry and Human Behavior, Warren Alpert Medical School of Brown University, Providence, RI, USA

3 Massachusetts Mental Health Center and the Department of Psychiatry, Harvard Medical School at Beth Israel Deaconess Medical Center, Boston, MA, USA

4 Department of Health Services, Policy and Practice, School of Public Health, Brown University, Providence, RI, USA

5 Department of Psychiatry, School of Medicine, University of California San Diego, San Diego, CA, USA 